Wagner Loesch VIANNA ${ }^{1}$ Marcos Eduardo PINESE ${ }^{1}$ Aline de Campos ROSSETO ${ }^{1}$ Cláudio Alvarenga de OLIVEIRA $^{1}$

Aníbal de Sant'Anna MORETTI ${ }^{1}$

\section{Correspondência para:}

WAGNER LOESCH VIANNA

Laboratório de Pesquisa em Suínos

Faculdade de Medicina Veterinária e

Zootecnia

Universidade de São Paulo

Rua Duque de Caxias Norte, 225

13630-000 - Pirassununga -SP

waloesch@fmvz.usp.br

Recebido para publicação: 12/03/2004 Aprovado para publicação: 12/12/2006

\title{
Indução da puberdade e sincronização do cio subsequente em leitoas pré-púberes utilizando gonadotrofinas exógenas
}

\author{
1 - Laboratório de Pesquisa em Suínos da Faculdade de Medicina \\ Veterinária e Zootecnia da Universidade de São Paulo, Pirassununga - SP
}

\section{Resumo}

A utilização de gonadotrofinas exógenas na indução da puberdade e sincronização do estro e ovulação é uma prática que influencia positivamente no manejo das marrãs, para a mais efetiva e econômica inclusão no plantel de matrizes em Sistemas Intensivos de Produção de Suínos. No entanto, poucos estudos têm sido desenvolvidos com a finalidade de avaliar o grau de sincronização do estro seguinte à indução da puberdade. Assim sendo, o objetivo do presente estudo foi, justamente, o de verificar a taxa de sincronização do estro subseqüente à puberdade induzida hormonalmente, e a comparação das características dos estros, induzido e subseqüente a este. Sessenta e seis leitoas pré-púberes tiveram a puberdade induzida através de combinação hormonal utilizando gonadotrofinas (eCG e LH) aos 145 dias de idade em média. Das 66 fêmeas que iniciaram o experimento, $35(53,03 \%)$ apresentaram cio até sete dias após a aplicação do LH, e $40(60,60 \%)$ das 66 fêmeas tiveram o estro subseqüente sincronizado, ou seja, até o $25^{\circ}$ dia após a aplicação do LH. Vinte e seis $(39,39 \%)$ fêmeas manifestaram o primeiro e segundo cios. As 26 $(39,39 \%)$ leitoas que não manifestaram o $2^{\circ}$ cio de maneira sincronizada foram abatidas, sendo que 15 apresentaram corpos lúteos e/ou corpos albicans e 11 não apresentaram as estruturas mencionadas anteriormente, sendo consideradas imaturas. Não houve diferença significativa da duração do cio na puberdade com o cio subseqüente ( $\mathrm{P}>0,05)$, tampouco da dispersão dos mesmos.

\section{Introdução}

$\mathrm{Na}$ produção de suínos em ciclo completo uma das fases que requer, atualmente, medidas específicas de manejo e cuidados especiais é a reposição de fêmeas. A idade à primeira monta ou Inseminação Artificial (IA) afeta diretamente a produtividade do rebanho, sendo essencial o conhecimento mais aprofundado da incorporação das fêmeas ao plantel de reprodutoras, ou seja, à reprodução. Dois fatores predominantes devem ser considerados quando envolvemos fêmeas jovens; o primeiro relaciona-se ao custo da alimentação durante o período nãoprodutivo, ou seja, da seleção à gestação e o reprodutivo ${ }^{1}$. segundo liga-se ao tempo de vida útil

Foxcroft e Aherne ${ }^{2}$ ressaltam a importância de se atingir a puberdade o mais precocemente, por volta dos 120 a 140 dias de idade, pois a estimulação precoce da marrã dá a oportunidade desta de experimentar número maior de ciclos estrais antes da cobrição, podendo interferir positivamente no tamanho da primeira leitegada e na própria vida útil reprodutiva. Atualmente a utilização de gonadotrofinas pode ser uma ferramenta útil para a indução da puberdade, pois promove uma sincronização do cio e da ovulação, facilitando o manejo de formação dos lotes de leitoas ${ }^{3}$. A eficácia da utilização hormonal 
prende-se ao tipo de combinação que traga o maior mimetismo do metabolismo hormonal endógeno, não havendo alterações significativas no desenvolvimento folicular.

Com o estro induzido sincronicamente, os produtores selecionam menos marrãs para reposição, adequando-as aos grupos de porcas já formados e comercializando o excedente de marrãs, a preço de mercado ${ }^{4}$. Assim, os dias não produtivos seriam diminuídos e os custos associados com fêmeas de reposição permaneceriam mais baixos para os sistemas de produção ${ }^{5}$.

Paterson $^{6}$ em sua revisão sobre a indução da puberdade em marrãs refere que a combinação mais comumente utilizada é a de 400 UI de PMSG e 200 UI de hCG, em uma única aplicação. Entretanto, a utilização da combinação hormonal de 600 UI de PMSG e $5 \mathrm{mg}$ de LH, em duas aplicações com intervalo de 72 horas, respectivamente, mostraram-se eficientes quanto à indução da puberdade e sincronização do estro em leitoas prépúberes $^{7,8,9}$.

Assim sendo, o objetivo do presente estudo foi o de analisar a sincronização do estro subseqüente à puberdade induzida hormonalmente, e a comparação das características dos estros, induzido e subseqüente a este.

\section{Materiais e Método}

Foram utilizadas 66 leitoas prépúberes Camborough $22^{\circledR}$ (Agroceres PIC), as quais foram alojadas em gaiolas individuais no setor de gestação do Laboratório de Pesquisa em Suínos FMVZ - USP, em Pirassununga. Aos 145,98 $\pm 10,32$ dias de idade e 75,39 $\pm 12,43 \mathrm{Kg}$ aplicou-se $600 \mathrm{UI}$ de eCG (Novormon ${ }^{\circledR}$, Syntex S. A., Argentina) e 72 horas depois $5 \mathrm{mg}$ de $\mathrm{LH}$ (Lutropin ${ }^{\circledR}$, Vetrepharm Canada Inc., Canadá).

Para a determinação do momento da ovulação no primeiro e segundo estros foram realizados exames ultra-sonográficos (Falco $100^{\circledR}$ transdutor linear convexo 3,5/
5,0 MHz, Pie Medical ${ }^{\circledR}$, Holanda) do ovário via transcutânea em períodos de 6 hs a partir de 24 hs da aplicação de LH, na indução da puberdade, e em períodos de $12 \mathrm{hs}$ a partir de 24 hs do início do estro subseqüente à indução da puberdade. O momento da ovulação foi determinado como sendo o valor médio entre o exame anterior à ovulação (quando ainda apresentava folículos) e o exame seguinte à ovulação (quando não se visualizava mais os folículos) em relação à aplicação do LH, na indução da puberdade, e em relação ao início do estro subseqüente.

$O$ início e o final do estro foi acompanhado através do passeio de dois machos adultos às 07:00 e 17:00, sendo o mesmo caracterizado pelo reflexo de tolerância ao homem na presença do macho. A verificação de estro foi realizada até o $25^{\circ}$ dia após a aplicação de LH, sendo as fêmeas que não apresentaram sinais característicos de estro até esta data, eram abatidas para o exame dos ovários e do útero.

Para a análise estatística foi utilizado o programa $\mathrm{SAS}^{10}$. A normalidade dos resíduos das variáveis intervalo LH-ovulação (IntLHOv); intervalo estro subseqüenteovulação (IntCioOv), duração do estro à puberdade (DurCioPub) e duração do estro subseqüente (DurCio) foram previamente analisadas através do PROC UNIVARIATE. A seguir as médias foram comparadas através da análise de variância. Foi utilizado o teste de Barttlet, para homogeneidade das variâncias, o qual comparou a dispersão do estro e das características, momento da ovulação da puberdade induzida e do estro subseqüente.

\section{Resultados e Discussão}

Das 66 fêmeas que iniciaram o experimento, $27(40,9 \%)$ manifestaram cio até quatro dias após a aplicação do LH, e 40 $(60,60 \%)$ das 66 fêmeas tiveram o estro subseqüente sincronizado, ou seja, até o $25^{\circ}$ dia após a aplicação do LH. Vinte e seis $(39,39 \%)$ fêmeas manifestaram o primeiro 
Tabela 1 - Dados de intervalo aplicação LH-ovulação (IntLHOv) e intervalo início do cio subseqüente-ovulação (IntCioOv). São Paulo, 2004

\begin{tabular}{cccc}
\hline Variável & $\mathrm{N}$ & Média & $\begin{array}{c}\text { Desvio } \\
\text { Padrão* }\end{array}$ \\
\hline IntLHOv & 40 & $36,08^{\mathrm{a}}$ & $3,99^{\mathrm{A}}$ \\
IntCioOv & 21 & $34,43^{\mathrm{a}}$ & $6,71^{\mathrm{A}}$ \\
\hline
\end{tabular}

$A, a$ Letras iguais na mesma coluna não diferem entre si $(P>0,05)$

* Teste de Bartlett para homogeneidade das variâncias

e segundo cios. As 26 (39,39\%) leitoas que não manifestaram o $2^{\circ}$ cio de maneira sincronizada foram abatidas, sendo que 15 apresentaram corpos lúteos e/ou corpos albicans e 11 não apresentaram as estruturas mencionadas anteriormente, sendo consideradas imaturas.

Avaliações foram realizadas ${ }^{7,8,9}$ quanto aos percentuais de fêmeas que manifestaram o cio, não manifestaram o cio, mas que apresentaram ovários com estruturas, corpos lúteos e/ou albicans, e ainda as que mostraram-se imaturas, ou seja, não apresentaram estruturas nos ovários. $\mathrm{O}$ seqüenciar dos referidos estudos demonstrou menor variação no percentual de manifestação de estro induzido em fêmeas pré-púberes, pela aplicação de combinação hormonal associado à presença do macho, em contraste com maior variação dos percentuais na sincronização em averiguações que empregaram somente a estimulação do macho ${ }^{11}$. As idades para indução hormonal da puberdade em marrãs foram aos 126 e 140 dias de idade, e ao abate (aproximadamente cinco dias após a ovulação) verificou-se a presença de cistos ovarianos que variavam de 8,7 a $68,0 \%$, na dependência do protocolo hormonal ${ }^{7,8}$.

Os cistos ovarianos podem levar o animal ao anestro ou simplesmente desuniformizar o lote sincronizado ${ }^{12}$, sendo esta a causa de algumas fêmeas não terem apresentado o segundo cio de maneira sincronizada, embora no momento do abate não tenha sido verificada a presença de cistos ovarianos.

Aliado à presença de cistos ovarianos, existem fêmeas que foram refratárias à
Tabela 2 - Dados de duração do cio da puberdade (DurCioPub) e Duração do cio subseqüente (DurCio). São Paulo, 2004

\begin{tabular}{cccc}
\hline Variável & N & Média & $\begin{array}{c}\text { Desvio } \\
\text { Padrão* }\end{array}$ \\
\hline DurCioPub & 35 & $43,88^{\text {a }}$ & $18,14^{\mathrm{A}}$ \\
DurCio & 40 & $53,94^{\mathrm{a}}$ & $14,41^{\mathrm{A}}$ \\
\hline
\end{tabular}

${ }^{A}, a$ Letras iguais na mesma coluna não diferem entre si $(P>0,05)$

* Teste de Bartlett para homogeneidade das variâncias

indução hormonal. Paterson e Lindsay (1981) relataram 15\% de fêmeas refratárias à indução hormonal. A imaturidade do eixo hipotálamo-hipófise-ovário, representada pelo reduzido número de receptores às gonadotrofinas, por exemplo, pode ser a causa desta não resposta à combinação hormonal. Foxcroft e Aherne ${ }^{2}$ sugerem o descarte das fêmeas que não respondem à indução da puberdade, sob o argumento de que apresentarão vida útil reduzida, com baixos índices de fertilidade.

Existe ainda, mesmo que de modo subjetivo, a possibilidade de algumas fêmeas apresentarem cio silencioso, onde os eventos hormonais do estro ocorrem, mas os sintomas externos de cio não se manifestam. Geralmente fêmeas jovens (leitoas e primíparas) apresentam maiores incidências de cio silencioso ${ }^{13,14}$. Algumas fêmeas apresentavam alguns sinais de cio, como edema de vulva, presença de muco cristalino na vulva, entretanto, não apresentavam o reflexo de tolerância ao macho, tampouco ao homem, prejudicando a determinação real do início de cio e, conseqüentemente, o esquema de inseminação artificial. A resposta à indução hormonal, com a manifestação de estro evidente, poderia ser associada às fêmeas que apresentassem uma maturidade sexual precoce, ou seja, fêmeas com uma vida útil reprodutiva mais prolongada, possivelmente, em comparação às que não mostrassem sinais evidentes de estro, sugerindo-se, nesse caso, uma maneira de se testar uma seleção de leitoas com maior precocidade sexual.

$\mathrm{Na}$ tabela 1 pode-se verificar que a média e a dispersão do momento da ovulação em relação à aplicação do LH (cio 
induzido) e ao início do cio (cio subseqüente à indução da puberdade) mantiveram-se iguais $(\mathrm{P}>0,05)$. Não foram encontrados, na literatura consultada, trabalhos que informassem o grau de sincronização do estro subseqüente à indução da puberdade. Outra particularidade na tabela 1 foi que mesmo havendo a manifestação de cio, alguns exames ultra-sonográficos de ovários não eram capazes de identificar a presença de folículos. Dessa maneira, apenas 21 fêmeas entraram na análise de variância, pois em 19 das 40 fêmeas cobertas não foi possível a visualização de folículos para determinação do momento da ovulação. A comparação do intervalo aplicação do LH-ovulação e do intervalo início do cio subseqüente à induçãoovulação somente foi possível devido o pico do $\mathrm{LH}$ ser coincidente com o início do estro (em média 5 hs antes do início do cio) ${ }^{15}$. As médias dos intervalos LH-ovulação e início do cio-ovulação foram semelhantes, não apresentando diferenças significativas ( $\mathrm{P}>0,05)$. Da mesma maneira com a dispersão dos intervalos, representada pelo desvio-padrão.

As durações médias de estro no cio induzido e no cio subseqüente foram iguais
( $\mathrm{P}>0,05)$, aferindo-se que há uma mimetização endocrinológica da combinação hormonal com o cio normal (Tabela 2). Há uma semelhança destes resultados com os obtidos em outros experimentos pelo Laboratório de Pesquisa em Suínos, onde Gama et al. ${ }^{8}$ e Carbone ${ }^{7}$ encontraram uma duração de cio e momento da ovulação após a aplicação do LH de aproximadamente 41 horas e 40 horas, respectivamente.

\section{Conclusões}

A combinação hormonal eCG mais LH foi efetiva na indução e sincronização da puberdade, havendo, no entanto, fêmeas que atingem a maturidade sexual, mas que não manifestam estro no período esperado, indicando provavelmente a presença de cio silencioso, e fêmeas refratárias ao tratamento hormonal. A característica momento da ovulação, em relação à aplicação do LH ou ao início do cio, manteve-se semelhante na indução hormonal da puberdade e no cio subseqüente, o que não aconteceu com a duração do estro, onde a utilização hormonal reduziu a sua duração.

\section{Induction of puberty and following estrus synchronization in prepubertal gilts using exogenous gonadotropins}

\footnotetext{
Abstract

The utilization of exogenous gonadotropins in the puberty induction and estrus/ovulation synchronization is a management that influence positively gilts handle, to the better effectiveness and economic inclusion of gilts in the Intensive Swine Production System herd. However, few researches have been developed to evaluate the subsequent estrus synchronization after puberty induction. Thus, the aim of this study was to verify the subsequent estrus synchronization after the hormonal puberty induction and to evaluate the inducted and the following one estrus characteristics. Sixty six prepubertal gilts had their puberty inducted by a hormonal combination using gonadotropins (eCG and LH) at 145 days old, in average. A total of $35(52,03 \%)$ of the 66 initially gilts showed estrus up to seven days after LH administration, and $40(60,60 \%)$ had the subsequent estrus synchronized (synchronization was defined as the gilts which showed estrus up to day 25 after LH administration). Twenty six gilts $(39,39 \%)$ showed the first and second estrus. The 26 $(39,39 \%)$ remaining gilts that didn't show the second estrus synchronized were slaughtered, which 15 presented corpus albicans
} 
and/or corpora lutea and 11 were considered immature because they didn't present any structure mentioned above in the ovaries. There was no significant difference between the puberty estrus duration and the subsequent estrus duration $(\mathrm{P}>0,05)$, neither their dispersion.

\section{Referências}

1 ROZEBOOM, D. W.; PETTIGREW, J. E.; MOSER, R. L.; CORNELIUS, S. G.; KANDELGY, S. M. Influence of gilt age and body composition at first breeding on sow reproductive performance and longevity. Journal of Animal Science, v. 74, p. 138-150, 1996.

2 FOXCROFT, G.; AHERNE, F. Manejo da marrã de reposição e da porca de primeiro parto: Parte II. Selecionando marrãs para uma melhor vida útil reprodutiva. In: SIMPÓSIO INTERNACIONAL DE REPRODUÇÃO E INSEMINAÇÃO ARTIFICIAL EM SUÍNOS, 7., 2000, Foz do Iguaçu. Anais... Foz do Iguaçu: Associação Internacional de Reprodução e Inseminação Artificial em Suínos, 2000. p. 91-98.

3 PRESSING, A. L. Pharmacologic control of swine reproduction. Veterinary Clinics of North America. Food Animal Practice, v. 8, n. 3, p. 707-723, 1992.

4 BRITT, J. H.; DAY, B. N.; WEBEL, S. K.; BRAUER, M. A. Induction of fertile estrus in prepubertal gilts by treatment with a combination of pregnant mare's serun gonadotropin and human chorionic gonadotropin. Journal of Animal Science, v. 67, p. 1148-1153, 1989.

5 KNOX, R. V.; TUDOR, K. W.; RODRIGUEZ-ZAS, S. L.; ROBB, J. A. Effect of subcutaneous vs intramuscular administration of PG600 on estrual and ovulatory responses of prepubertal gilts. Journal of Animal Science, v. 78, p. 1732-1737, 2000.

6 PATERSON, A. M. The controled induction of puberty. In: COLE, D. J. A.; FOXCROFT, G. R. (Ed.). Control of Pig Reproduction. London: Butterworths, 1982, p. 139-159.

7 CARBONE, A. Emprego de gonadotrofinas exógenas na indução e sincronização da puberdade em marrãs. 2002. 60 f. Dissertação (Mestrado em Reprodução Animal) - Faculdade de Medicina Veterinária e Zootecnia, Universidade de São Paulo, São Paulo, 2002.

8 GAMA, R. D.; VIANNA, W. L.; MORETTI, A. S.; CARBONE, A.; PORTELLA, G.; CANDINI, P. H.; VIANA, C. H. C.; PINESE,M. A.; ROSSETO, A. C.; LAGO, V. Avaliação da utilização do LH porcino (Lutropinâ) para indução e sincronização da puberdade em fêmeas suínas. In: CONGRESSO LATINO AMERICANO DE SUINOCULTURA, 1., 2002, Foz do Iguaçu. Anais... Foz do Iguaçu: Associação Latinoamericana de Suinocultura, 2002. p. 149-150.

9 LAGO, V. Estudo dos efeitos combinados de gonadotrofinas e flushing em marrãs à puberdade. 2003. 87 f. Dissertação (Mestrado em Medicina Veterinária) - Faculdade de Medicina Veterinária e
Zootecnia, Universidade de São Paulo, Pirassununga, 2003.

10 SAS. SAS STATS user's guide: SAS, versão 8.2. Cary, 2001. 1 CD-ROM.

11 MUNIZ, A.; MORETTI, A. S.; RODRIGUES, P. H. M. Efeito da presença do macho na indução e duração do estro em marrãs, submetidas à alimentação, à vontade e restrita. In: CONGRESSO LATINO AMERICANO DE SUINOCULTURA, 1., 2002, Foz do Iguaçu. Anais... Foz do Iguaçu: Associação Latinoamericana de Suinocultura, 2002

12 RYAN, P. L.; RAESIDE, J. I. Cystic ovarian degeneration in pigs: a review. Irish Veterinary Journal, v. 44, p. 22-25, 1991.

13 SILVEIRA, P. R. S.; WENTZ, I.; BARROS, S. S. Leitoas que não entram em cio: observações do trato genital após o descarte. Comunicado técnico EMBRAPACNPSA, n. 88, p. 1-2, 1985.

14 SILVEIRA, P. R. S.; WENTZ, I. Anestro e cio silencioso em porcas desmamadas. Comunicado técnico EMBRAPA-CNPSA, n. 104, p. 1-2, 1986.

15 VAN RENS, B. T. T. M.; HAZELEGER, W.; VAN DER LENDE, T. Periovulatory hormone profiles and components of litter size in gilts with different estrogen receptor (ESR) genotypes. Theriogenology, v. 53, p. 1375-1387, 2000. 